\title{
Potential short-term outcome of an uncontrolled COVID-19 epidemic in Lombardy, Italy, February to March 2020
}

Giorgio Guzzetta ${ }^{1}$, Piero Poletti ${ }^{1}$, Marco Ajelli ${ }^{1}$, Filippo Trentini ${ }^{1}$, Valentina Marziano ${ }^{1}$, Danilo Cereda ${ }^{2}$, Marcello Tirani $^{2,3}$,

Giulio Diurno ${ }^{2}$, Annalisa Bodina ${ }^{2}$, Antonio Barone ${ }^{2}$, Lucia Crottogini2 ${ }^{2}$, Maria Gramegna² , Alessia Melegaro ${ }^{4,5}$, Stefano Merler ${ }^{1,5}$

1. Bruno Kessler Foundation, Trento, Italy

2. Lombardy Region, Directorate General for Health, UO Prevenzione, Milan, Italy

3. Health Protection Agency of Pavia, Department of Hygiene and Preventive Medicine, Pavia, Italy

4. Bocconi University, Dondena Centre for Research on Social Dynamics and Public Policy, Milan, Italy

5. These authors are joint senior authors and contributed equally to this work.

Correspondence: Alessia Melegaro (alessia.melegaro@unibocconi.it)

Citation style for this article:

Guzzetta Giorgio, Poletti Piero, Ajelli Marco, Trentini Filippo, Marziano Valentina, Cereda Danilo, Tirani Marcello, Diurno Giulio , Bodina Annalisa, Barone

Antonio, Crottogini Lucia, Gramegna Maria, Melegaro Alessia, Merler Stefano. Potential short-term outcome of an uncontrolled COVID-19 epidemic in Lombardy, Italy, February to March 2020. Euro Surveill. 2020;25(12):pii=2000293. https://doi.org/10.2807/1560-7917.ES.2020.25.12.2000293

Sustained coronavirus disease (COVID-19) transmission is ongoing in Italy, with 7,375 reported cases and 366 deaths by 8 March 2020. We provide a modelbased evaluation of patient records from Lombardy, predicting the impact of an uncontrolled epidemic on the healthcare system. It has the potential to cause more than 250,039 (95\% credible interval (Crl): $147,717-459,890)$ cases within 3 weeks, including 37,194 (95\% Crl: 22,250-67,632) patients requiring intensive care. Aggressive containment strategies are required.

On 20 February 2020, a case of coronavirus disease (COVID-19) was notified in Lombardy, Italy, uncovering ongoing transmission in at least two other regions (Emilia Romagna and Veneto) [1]. To rapidly assess risks of the current situation, we analysed the line list of reported cases in Lombardy to project the number of cases, should the epidemic be left uncontrolled.

\section{Epidemic projections}

We projected the number of COVID-19 cases in 593 municipalities of Lombardy where at least one case of community transmission (i.e. excluding cases in healthcare workers or known nosocomial exposure) had been recorded by 8 March 2020 . These represented $39.4 \%$ of all municipalities in the region and a total population of ca 6.9 million inhabitants $(68.8 \%$ of the Lombardy population). The projections were based on a stochastic susceptible-infectious-removed (SIR) transmission dynamic model for each municipality and assuming that no control measures were in place. The model considered a population structured in 20 age groups (19 5-year age groups from o to 94 years and one age group for $\geq 95$-year-olds) according to municipality-specific age distributions [2]. The Polymod contact matrix for Italy was incorporated to simulate the heterogeneity of contacts by age [3]. The model considered three consecutive infectious compartments to simulate a gamma distributed generation time of a mean of 6.6 days [1], longer than estimates obtained for Chinese provinces outside Hubei [4]. $R_{0}$ was sampled from the posterior distribution estimated for Lombardy: mean: 3.1, $95 \%$ credible interval (Crl): 2.9-3.2 [1]. We assumed asymptomatic and symptomatic individuals to be equally infectious as shown by preliminary analysis of virological data from the same region [1].

We considered age-specific reporting and severity rates, which were estimated from the Lombardy line list. In particular, the fraction of reported infections at different ages $r(a)$ was defined as

$$
r(a)=r_{m} \frac{p(a)}{i(a)}
$$

where $r_{m}$ represents the overall fraction of reported infections in the population; $i(a)$ is the proportion of expected infections in age $a$ and $p(a)$ is the proportion of cases reported in age group $a$. We computed $i(a)$ as the average age distribution of cases at the end of 500 simulated epidemics in the model.

Note that $r(a)$ represents the probability that an infection occurring in age $a$ is reported, while $p(a)$ represents the contribution of reported cases of age $a$ to the overall amount of reported cases. We estimated $p(a)$ for seven age groups (0-19, 20-29, 30-39, 40-49, 50-59, 50-69 and $\geq 70$ years). Only seven age groups were 


\section{FIGURE 1}

Observed and estimated cumulative number of reported coronavirus disease cases aggregated over 593 municipalities (population 6.9 million), Lombardy, 1-27 February 2020 ( $n=1,400$ observed cases)

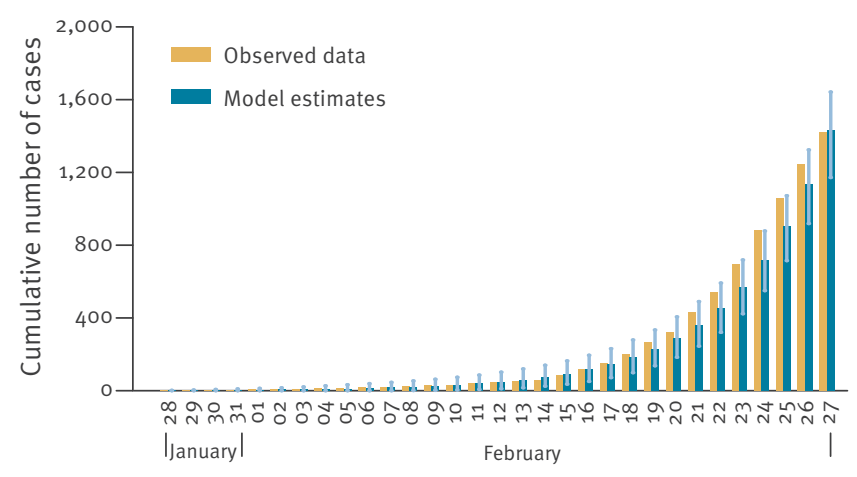

Date in 2020

Vertical lines represent $95 \%$ credible intervals of model estimates.

considered here in order to analyse a sufficiently large sample of reported cases for each age group. Estimates of $p(a)$ were obtained by a Markov chain Monte Carlo (MCMC) sampling, applied to the multinomial likelihood of observing the reported number of cases $C(a)$ in age group (a):

$$
L=\frac{C !}{\prod_{a} C(a) !} \prod_{a} p(a)^{C(a)}
$$

where $C$ is the overall number of positive individuals. Specifically, the posterior distribution of $p(a)$ was computed by using the random-walk MetropolisHastings algorithm based on normal jump distribution, which represents a common method to generate sequence of samples from an unknown probability distribution [5].

The relative risk of developing critical disease by age, $z(a)$, was assumed to be proportional to the fraction of critical and deceased cases observed in the corresponding age group, $v(a)$ :

$$
z(a)=\frac{\nu(a)}{\sum_{a} p(a) \nu(a)}
$$

The proportion of reported cases of age $a$ who develop critical disease, $w(a)$, is given by $w(a)=w_{m} z(a)$, where $w_{m}$ represents the overall fraction of critical cases among reported cases. In the projections, we assumed $r_{m}=9.2 \%$ (95\% Crl: 5-20\%) for the overall reporting rate [6] and $w_{m}=15 \%$ for the overall proportion of reported cases requiring intensive care [1].

For each municipality, we initialised the model with a fully susceptible population and a single infectious individual, of age chosen with probability proportional to its age structure. Since we do not know when the initial infection was introduced in each municipality, we start our model with one infectious individual at day 1 of the simulation time. At a given day $t$ of the simulation time, the cumulative number of confirmed cases in the model reaches the cumulative number of confirmed cases observed on 27 February in that municipality. We then assumed that day $t$ of the simulation time corresponds to the calendar day 27 February, dated back the day of first introduction accordingly and projected the number of cases between 9 and 28 March 2020. Data reported in the line list after 27 February may still be incomplete because of reporting delays [1]. We filtered out all simulations that ended in stochastic extinction of the epidemic before 27 February and we ran the model until we had 500 valid simulations for each municipality. A validation of the model is shown in Figure 1.

The observed and modelled age distribution of reported and critical cases is shown in Figure 2. About $63 \%$ (95\% Crl: 60-66\%) of critical cases were estimated to be individuals older than 70 years. In the absence of any intervention, we estimated that the number of new cases per day during the period 9-28 March would reach 19,060 (95\% Crl: 9,898-41,491). A total of 250,039 ( $95 \% \mathrm{Crl}: 147,717-459,890)$ reported cases were expected to occur between 9 and 28 March in the considered population of Lombardy (Figure 3). During the same period, the model estimated a total of 37,194 (95\% Crl: $22,250-67,632)$ patients requiring critical care. As a comparison, the total number of COVID-19 cases recorded in the Lombardy line list by 23 March 2020 (in the presence of interventions) was $28,761,20,043$ of whom were symptomatic cases.

\section{Discussion}

Following the notification of a COVID-19 case in Lombardy on 20 February 2020, which triggered testing and detection of autochthonous transmission in at least two other regions (Emilia Romagna and Veneto), immediate control measures were adopted by regional and national governments, including the institution of quarantined zones on 23 February enclosing more than 50,000 inhabitants. Despite these measures, the spatial distribution of the epidemic expanded rapidly throughout the country. By 8 March, all Italian regions had reported at least one case, with seven regions reporting more than 100 cases and nine regions reporting at least one death. In Lombardy, the regional health authority started to fill in a line list of COVID-19-positive cases [1], comprising 5,830 infections, with 5,626 confirmed symptomatic cases and 588 infections recorded among healthcare workers as at 8 March.

The ongoing COVID-19 outbreak in Italy and, particularly, in the Lombardy region has put the healthcare system under massive strain, given the rapid epidemic spread and the need to provide intensive care support to an increasing number of patients. Healthcare systems and policymakers had to face rapid decisions 


\section{FIGURE 2}

Age distribution of reported cases $(n=1,400)$ and of patients either deceased or requiring intensive care $(n=278)$, compared with corresponding model estimates, Lombardy line list, 1-27 February 2020

A. Reported cases vs corresponding model estimates

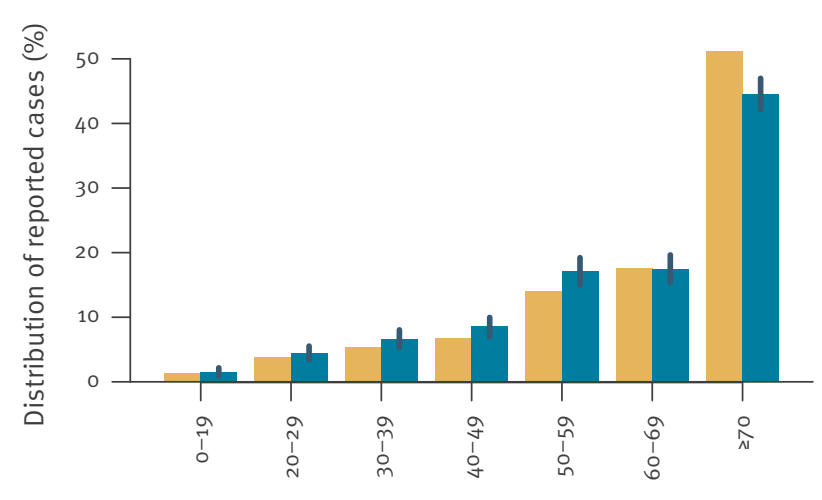

Age (years)

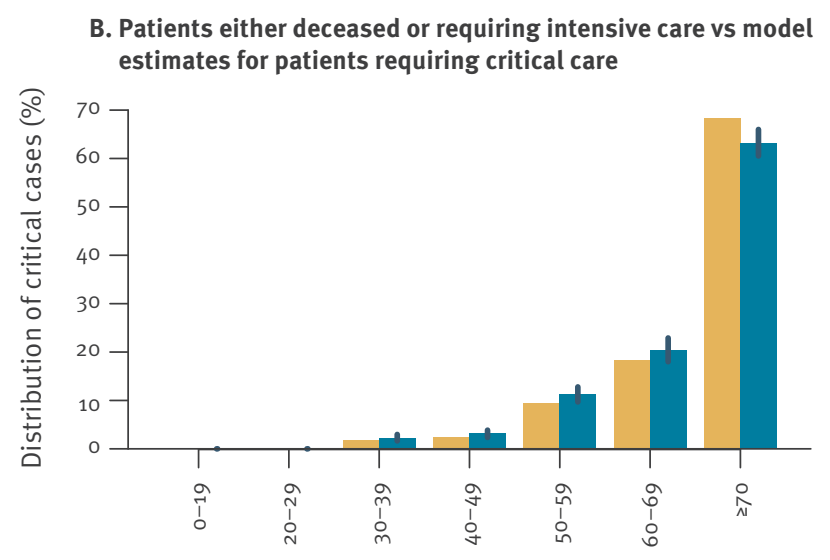

Age (years)

Observed data Model estimates

Vertical lines represent $95 \%$ credible intervals of model estimates.

\section{FIGURE 3}

Projected daily number of reported and critical cases, aggregated over 593 municipalities, Lombardy, 9 and 28 March 2020

A. Reported cases

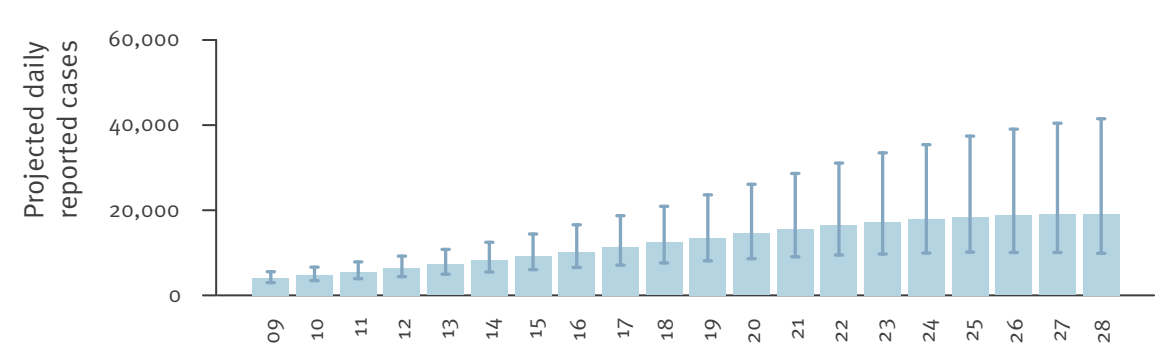

March
B. Critical cases, by age group

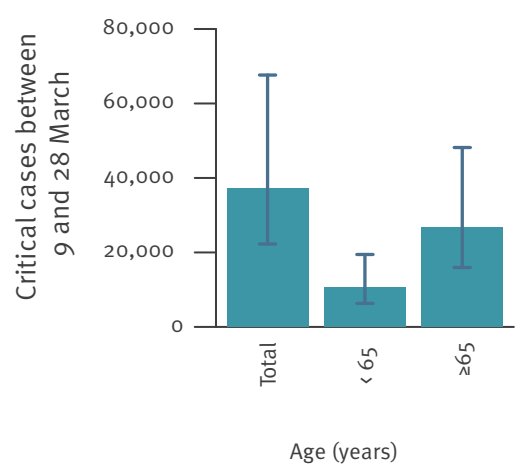

Date in 2020

Bars: mean estimates; vertical lines: $95 \%$ of credible interval associated with uncertainties in values of $\mathrm{R}_{0}$, the overall reporting rate $r_{m}$, as well as the variability of the stochastic model realisations.

to contain the contagion process and limit the number of fatalities. Here we presented epidemic projections assuming no interventions and $R_{0}=3.1$. This value of the basic reproduction number was estimated in Cereda et al. [1], analysing the exponential phase of the epidemic in Lombardy; it compares well to the estimates obtained during the early epidemic phase in Wuhan, China [7].

The still limited knowledge on COVID-19 epidemiology in Italy certainly represents a limitation of this study as some parameters such as the overall reporting rate and the overall proportion of reported cases requiring intensive care were estimated in the Chinese context $[4,6]$. Based on the analysis of Chinese contact data, we considered that children are as likely to be infected as adults [8,9]. Moreover, we assumed no differences in infectiousness between symptomatic and asymptomatic infected individuals. Although this choice was supported by the analysis of virological data presented by Cereda et al. [1], further evidence is required to fully validate this hypothesis. Finally, we did not consider potential temporal trends in the reporting rate and delays in the development of symptoms requiring intensive care. It is also possible that our projections underestimate the potential impact of an uncontrolled 
epidemic, as we do not allow for contacts between individuals in different municipalities (which may reignite transmission in locations where the infection becomes stochastically extinct) and we assumed that the infection did not spread further to other municipalities inside and outside Lombardy.

Even in the presence of the above limitations, the simulated cumulative number of cases between 28 January and 27 February well compares with data (for each considered day, data points fall in the credible interval of model estimates). The presented results show that in a scenario of uncontrolled transmission, the number of severe and critical cases will become largely unsustainable for the healthcare system in a very short time. Drastic governmental interventions and widespread behavioural changes of the population are required to limit COVID-19 transmission and avoid catastrophic effects on the healthcare system.

\section{Acknowledgements}

H2020 Mood Project.

\section{Conflict of interest}

None declared.

\section{Authors' contributions}

GG conceived of the study, performed the analysis, contributed to interpret results, drafted the manuscript. PP performed the analysis, contributed to interpret results, drafted the manuscript. MA conceived of the study, contributed to interpret results, drafted the manuscript. FT performed the analysis, contributed to interpret results, drafted the manuscript. VM performed the analysis, contributed to interpret results, drafted the manuscript. DC contributed to interpret results,

MT contributed to interpret results, GD data management, contributed to interpret results, $\mathrm{AB}$ o contributed to interpret results, $A B$ a contributed to interpret results, $L C$ contributed to interpret results, MG contributed to interpret results, $A M$ performed the analysis, contributed to interpret results, drafted the manuscript. SM conceived of the study, contributed to interpret results, drafted the manuscript.

\section{References}

1. Cereda D, Tirani M, Rovida F, Demicheli V, Ajelli M, Poletti P, et al. The early phase of the COVID-19 outbreak in Lombardy, Italy. 23 Mar 2020.Available from: https://arxiv.org/ abs/2003.09320

2. Italian National Institute of Statistics (ISTAT). Popolazione residente per età, sesso e stato civile al $1^{\circ}$ Gennaio 2019. Rome: ISTAT. [Accessed 1 Mar 2020]. Available from: http:// demo.istat.it/pop2019/index.html

3. Mossong J, Hens N, Jit M, Beutels P, Auranen K, Mikolajczyk $\mathrm{R}$, et al. Social contacts and mixing patterns relevant to the spread of infectious diseases. PLoS Med. 2008;5(3):e74. https://doi.org/10.1371/journal.pmed.0050074 PMID: 18366252

4. Zhang J, Litvinova $M$, Wang W, Wang $Y$, Deng X, Chen X, et al Evolving epidemiology of novel coronavirus diseases 2019 and possible interruption of local transmission outside Hubei Province in China: a descriptive and modeling study. medRxiv
2020.02.21.20026328. https://doi.org/10.1101/2020.02.21.20 026328

5. Cauchemez S, Carrat F, Viboud C, Valleron AJ, Boëlle PY. A Bayesian MCMC approach to study transmission of influenza: application to household longitudinal data. Stat Med. 2004;23(22):3469-87. https://doi.org/10.1002/sim.1912 PMID: 15505892

6. Nishiura H, Kobayashi T, Yang Y, Hayashi K, Miyama T, Kinoshita R, et al. The rate of under ascertainment of novel coronavirus (2019-nCoV) infection: Estimation using Japanese passengers data on evacuation flights. J Clin Med. 2020;9(2):E419. https://doi.org/10.3390/jcm9020419

7. Li Q, Guan X, Wu P, Wang X, Zhou L, Tong Y, et al. Early transmission dynamics in Wuhan, China, of novel coronavirusinfected pneumonia. N Engl J Med. 2020;NEJMoa2001316. https://doi.org/10.1056/NEJMoa2001316 PMID: 31995857

8. Wu Z, McGoogan JM. Characteristics of and important lessons from the coronavirus disease 2019 (COVID-19) outbreak in china: summary of a report of 72314 cases from the Chinese Center for Disease Control and Prevention. JAMA. 2020. [Epub ahead of print] https://doi.org/10.1001/jama.2020.2648 PMID: 32091533

9. Bi Q, Wu Y, Mei S, Ye C, Zou X, Zhang Z, et al. Epidemiology and transmission of COVID-19 in Shenzhen China: analysis of 391 cases and 1,286 of their close contacts. medRxiv 2020.03.03.20028423; https://doi. org/10.1101/2020.03.03.20028423

\section{License, supplementary material and copyright}

This is an open-access article distributed under the terms of the Creative Commons Attribution (CC BY 4.0) Licence. You may share and adapt the material, but must give appropriate credit to the source, provide a link to the licence and indicate if changes were made.

Any supplementary material referenced in the article can be found in the online version.

This article is copyright of the authors or their affiliated institutions, 2020. 